\title{
The Reliability and Accuracy of Knee Implants Sizing Predicted by Digital Templating
}

\author{
Sergejs Zadoroznijs ***, Konstantins Kalnberzs */** \\ *University of Latvia \\ **Hospital of Traumatology and Orthopaedics, Riga, Latvia
}

\begin{abstract}
Summary
Introduction. Osteoarthritis of the knee is a common and frequently symptomatic illness. Total knee replacement (TKR) has evolved as an accepted, cost-effective and efficacious treatment modality for osteoarthritis and other forms of arthritic conditions of the knee joint. Preoperative planning is an important part of the surgical procedure. The inability to accurately determine the magnification factor of the radiograph is one of the major problems in analog preoperative planning of TKR. With the use of calibration objects, the digital images can be corrected for the magnification factor.
\end{abstract}

Aim of the Study. We aimed to determine the reliability and accuracy of digital templating in the pre-operative work-up for TKR.

Materials and Methods. A retrospective study was done in 105 caucasian adults, who had osteoarthritis of the knee. Digital templating was performed using a calibrating 25-mm metallic ball and Agfa Orthopaedic Tools digital software package by a surgeon not involved with the operation, who was blinded to the size of the implant inserted. The Press Fit Condylar Sigma Knee system was used in all the patients. Digital anteroposterior and lateral radiographs of the knee were used in measuring the implant size. The results from digital images were compared with the size of actual femoral and tibial implants used at the time of surgery.

Results. The correct size of the implant was predicted in 73 of $105(69,5 \%)$ of the femoral and 70 of $105(66,7 \%)$ of the tibial components. The correct size of the whole system was predicted in 58 of $105(55,2 \%)$ cases. The digital preoperative planning predicted 104 of $105(99,0 \%)$ femoral and tibial implants and 103 of 105 (98,1\%) whole systems to within one size.

Conclusions. We conclude that digital templating using a calibrating $25-\mathrm{mm}$ metallic ball and Agfa Orthopaedic Tools digital software is a reliable method of predicting the implant to within one size.

Key words: osteoarthritis of the knee, total knee replacement, digital templating, knee implants sizing

\section{INTRODUCTION}

Osteoarthritis is the result of mechanical and biological events that destabilize the normal processes of degradation and synthesis of articular cartilage chondrocytes, extracellular matrix, and subchondral bone. These changes include increased water content, decreased proteoglycan content, and altered collagen matrix, all leading to the deterioration of articular cartilage. (13)

Osteoarthritis of the knee is a common and frequently symptomatic illness. Its prevalence increases with age, from negligible in those aged 25-34 years to 20-40 per cent in those aged 75 and older. (5)

This number would certainly increase with extended longevity of patients. Severe osteoarthritis of knee joint is a common problem in older people and a major concern for pain and disability. Most patients with osteoarthritis of the knee are able to manage their symptoms with medical treatment and conservative methods, but a large number of patients referred to the specialist surgeon for further management have debilitating disease. (3)

Total joint replacement can be considered as the best end point for clinical trials evaluating disease-modifying osteoarthritis drugs. Great efforts are being made to validate a composite index, which could define states of severity and "need for total joint replacement." (7) Many parameters other than the severity of the disease itself influence the decision for surgery, however, including socioeconomic factors and access to health services. $(6 ; 11 ; 14)$

Total knee replacement (TKR) has evolved as an accepted, cost-effective and efficacious treatment modality for osteoarthritis and other forms of arthritic conditions of the knee joint.

Approximately 30,000 TKRs were carried out in 2000/01 in England and Wales (3). The total number of TKRs performed in UK has risen by over 20,000 between the years 2002 and 2004.

The demand for TKR is increasing mainly because of longer life expectancies and rising public expectations for quality of life and mobility in later years. Currently, approximately $2 \%$ of the population of 55 years age and above are so disabled that they need TKR, and this rate increases with age. The estimated prevalence in women is nearly twice as high as in men. (15)

TKR rate is increasing not only in UK but also all over the world (Table 1). (1)

TKR is indicated for pain relief and functional improvement in severe knee joint degeneration and arthritis. The goals of TKR surgery include adequate alignment of the prosthesis components and the limb, stability of the knee, and attainment of sufficient range of motion, which permits adequate movement to attain improved quality of life. (4)

Preoperative planning is an important part of the surgical procedure. The technical goals of preoperative 
planning of the TKR are to achieve accurate prosthetic seating with proper axial alignment. (10) Preoperative planning provides the surgeon with a tool in order to ascertain that the correct prosthetic component sizes are available. The inability to accurately determine the magnification factor of the radiograph is one of the major problems in analog preoperative planning of TKR. In addition, the use of templates with standard magnifications does not permit accurate correction of the magnification factor. (8)

For the TKR, the analog plans scored poorly concerning exact agreement. Even when allowing for one size difference, the results were disappointing. The digital plans for both components scored better, with more than 50\% exact agreements and more than 90\% agreements when allowing an error of one component size. The absolute differences between the sizes planned preoperatively for the TKR and implanted component sizes were significantly less for digital planning than for analog planning, regarding both the femoral component (mean difference 0.6; $p<0.001$ ) and the tibial component (mean difference $1.1 ; \mathrm{p}<0.001$ )

With the use of calibration objects, the digital images can be corrected for the magnification factor. This is generally assumed to be an advantage, but if the position of the calibration object differs too much from the region of interest, it will lead to a structural error in digital correction of magnification. In $95 \%$ of cases, variability in positioning of the calibration object can be expected to result in an error of correction of the magnification ranging from $-3 \%$ to $+3 \%$. Regarding analog plans for knee prostheses, an actual systematic error in planning seems plausible. (9)

\section{AIM OF THE STUDY}

We aimed to determine the reliability and accuracy of digital templating in the pre-operative work-up for TKR.

\section{MATERIALS AND METHODS}

A retrospective study was done to assess the accuracy of the knee implant sizing predicted by digital images in 105 caucasian adults, who had osteoarthritis of the knee. Digital templating was performed using a calibrating 25-mm metallic ball and Agfa Orthopaedic Tools digital software package by a surgeon not involved with the operation, who was blinded to the size of the implant inserted. The Press Fit Condylar Sigma Knee system was used in all the patients. Digital anteroposterior and lateral radiographs of the knee were used in measuring the implant size. The results from digital images were compared with the size of actual femoral and tibial implants used at the time of surgery.

\section{RESULTS}

The correct size of the implant was predicted in 73 of $105(69,5 \%)$ of the femoral and 70 of $105(66,7 \%)$ of the tibial components. The correct size of the whole system was predicted in 58 of $105(55,2 \%)$ cases. The digital preoperative planning predicted 104 of 105 $(99,0 \%)$ femoral and tibial implants and 103 of 105
$(98,1 \%)$ whole systems to within one size. There were 2 cases in which the predicted implant ( 1 case - femoral, other - tibial) appeared to be undersized from the final component by 2 sizes. The tibial component appeared to be more often undersized- 25 of $105(23,8 \%)$ versa 22 of $105(20,9 \%)$ in femoral component. The rate of femoral and tibial components to be oversized on the preoperative radiographs appeared to be the same - 10 of $105(9,5 \%)$. There were no cases of components to be oversized by 2 sizes.

\section{DISCUSSION}

Not many studies on the reliability and accuracy of knee implants sizing predicted by digital templating can be found up to date and their data is very different.

In this retrospective study we demonstrated our data and tried to compare it with several identical studies. (Table 2)

We can see that the data for exactly predicting femor or tibia size is of a very wide range - from $53,1 \%$ to $82,5 \%$ in femor and from $59,3 \%$ to $79,5 \%$ in tibia. In our study digital templating was performed by one of the authors - a surgeon not involved with the operation, who was blinded to the size of the implant inserted. There is no data if the templating surgeon was involved in the operation and thus influencing the choice of the size of the implants in the other studies, so we cannot assume that our result in exactly predicting the implant is really inferior to the one of the third study.

The data for predicting femor or tibia to within one size is very similar in all studies and is not less than $93,0 \%$, which is a very good result. We predicted femor or tibia to within one size in $99,0 \%$, which is the highest result from the studies compared.

There was a trend toward implants to be undersized in digital templating in our study, which can be explained by the will of the templating surgeon to select the implant not overhanging the bone and by the desire of the operating surgeon to preserve as much bone as possible.

Future prospective studies are needed to determine whether preoperative digital templating by the operating surgeon impacts his choice thus improving the accuracy of knee implants sizing.

\section{CONCLUSIONS}

We conclude that digital templating using a calibrating 25-mm metallic ball and Agfa Orthopaedic Tools digital software is a reliable method of predicting the implant to within one size.

\section{REFERENCES}

1. Adravanti P. The socioeconomic challenge of the arthritic knee epidemiology // The First Open Meeting of the European Knee Associates, The Osteoarthritic Knee. Vienna, Austria : 2011

2. Del Gaizo D, Soileau ES, Lachiewicz PF. Value of preoperative templating for primary total knee arthroplasty // J Knee Surg, 2009; 22(4):284-293 
3. Department of Health (available to Health Services Research Collaboration) 2000/01. Hospital Episode Statistics for England and Wales. London: Department of Health, 2001.

4. Dorr LD, Boiardo RA. Technical considerations in total knee arthroplasty // Clin Orthop Relat Res, 1986; 205:5-1 1

5. Felson DT. Epidemiology of hip and knee osteoarthritis // Epidemiologic Reviews, 1998; $10: 1-28$

6. Fielden JM, Cumming JM, Horne JG, et al: Waiting for hip arthroplasty: Economic costs and health outcomes // J Arthroplasty, 2005; 20:990-997

7. Gossec L, Hawker G, Davis AM, et al: OMERACT/ OARSI initiative to define states of severity and indication for joint replacement in hip and knee osteoarthritis // J Rheumatol, 2007; 34:1432-1435

8. Heal J, Blewitt N. Kinemax total knee arthroplasty: trial by template // J Arthroplasty, 2002; 17: 90-94

9. Jamali AA. Digital Templating and Preoperative Deformity AnalysiswithStandardImaging Software// Clin Orthop Relat Res, 2009; 467:2695-2704

10. Krackow KA. Total knee arthroplasty: technical planning and surgical aspects // Instr Course Lect, 1986; 35: 272-282

11. March L, Cross M, Tribe K, et al: Cost of joint replacement surgery for osteoarthritis: The patients' perspective. J Rheumatol, 2002; 29:1006-1014.

12. Specht LM, Levitz S, Iorio R, Healy WL, Tilzey JF. A comparison of acetate and digital templating for total knee arthroplasty // Clin Orthop Relat Res, 2007; 464:179-183

13. ST Canale, JH Beaty. Miscellaneous Nontraumatic Disorders // ST Canale, JH Beaty. Campbell's Operative Orthopaedics, 11 th Edition. Philadelphia, PA, USA: Mosby Elsevier; 2007

14. Steel N, Melzer D, Gardener E, et al: Need for and receipt of hip and knee replacement-a national population survey // Rheumatology (Oxf), 2006; 45:1437-1441.

15. Tennant A, Fear J, Pickering A, Hillman M, Cutts A, Chamberlain MA. Prevalence of knee problems in the population aged 55 years and over: identifying the need for knee arthroplasty // BMJ, 1995; 310:1291-1293

16. Wongsak S, Kawinwonggowit V, Mulpruck P, Channoom T, Woratanarat P. Accuracy of knee implants sizing predicted by digital images // J Med Assoc Thai, 2009; 92 Suppl 6:S85-90

\section{Address:}

Sergejs Zadorožnijs

Hospital of Traumatology and Orthopaedics

Duntes street 22

Riga, LV-1005

Latvia

E-mail: szador12de@gmail.com
Table 1. Annualized growth in TKR procedures all over the world

\begin{tabular}{|c|c|c|c|}
\hline Country & $\begin{array}{c}\text { Years of } \\
\text { available TKR } \\
\text { data }\end{array}$ & $\begin{array}{c}\text { Annualized } \\
\text { growth } \\
\text { in TKR } \\
\text { procedures }\end{array}$ & $\begin{array}{c}\text { Annualized } \\
\text { growth in } \\
\text { procedures } \\
\text { rate / } 10^{5}\end{array}$ \\
\hline Australia & $2003-2008$ & $6,7 \%$ & $5,0 \%$ \\
\hline Canada & $2002-2008$ & $10,3 \%$ & $9,1 \%$ \\
\hline Finland & $1997-2009$ & $7,2 \%$ & $6,9 \%$ \\
\hline France & $2002-2007$ & $5,3 \%$ & $3,6 \%$ \\
\hline Germany & $2005-2008$ & $6,9 \%$ & $7,1 \%$ \\
\hline Italy & $1999-2008$ & $12,8 \%$ & $12,2 \%$ \\
\hline Netherlands & $1997-2007$ & $9,4 \%$ & $8,8 \%$ \\
\hline Portugal & $1997-2008$ & $17,0 \%$ & $16,6 \%$ \\
\hline Spain & $1997-2008$ & $11,5 \%$ & $10,1 \%$ \\
\hline Switzerland & $1998-2008$ & $14,7 \%$ & $14,0 \%$ \\
\hline USA & $1997-2008$ & $7,9 \%$ & $6,8 \%$ \\
\hline
\end{tabular}

Table 2. Comparison of different studies' data

\begin{tabular}{|c|c|c|c|c|}
\hline & $\begin{array}{c}\text { Exact } \\
\text { femoral } \\
\text { size }\end{array}$ & $\begin{array}{c}\text { Femor } \pm \\
\text { l size }\end{array}$ & $\begin{array}{c}\text { Exact } \\
\text { tibial } \\
\text { size }\end{array}$ & $\begin{array}{c}\text { Tibia } \pm \\
\text { l size }\end{array}$ \\
\hline $\mathbf{1}^{\text {st }}$ study (16) & $53,1 \%$ & $97,6 \%$ & $59,3 \%$ & $95,1 \%$ \\
\hline $\mathbf{2}^{\text {nd }}$ study (12) & - & $93,0 \%$ & - & $93,0 \%$ \\
\hline $3^{\text {rd }}$ study (2) & $82,5 \%$ & $97,0 \%$ & $79,5 \%$ & $92,5 \%$ \\
\hline Our study & $69,5 \%$ & $99,0 \%$ & $66,7 \%$ & $99,0 \%$ \\
\hline
\end{tabular}

